\title{
Los bloqueos de escritura. en la elaboración de las tesis doctorales
}

\author{
José Matías Albarrán \\ Universidad de Los Andes, Venezuela \\ osematiasap@gmail.com \\ Karen Yeniree Uzcátegui \\ Universidad de Los Andes, Venezuela \\ kyul2886@gmail.com
}

Recibido: 09-06-2020 / Aceptado: 18-10-2020

\section{Resumen}

Este estudio de casos se aproxima a la comprensión de los bloqueos de escritura que experimentan los doctorandos en la elaboración de su tesis. Se describen y analizan las situaciones en que incurren los tesistas durante el proceso de escritura, para luego ofrecer un marco interpretativo de los factores que inciden en los bloqueos. Se entrevistó, incidentalmente, a siete cursantes de un doctorado venezolano. Como resultado se observó que los tesistas presentan obstáculos de naturaleza cognitiva, conductual, pedagógica, afectiva y motivacional que los bloquean. Se concluye que es importante ofrecer supervisión, motivación y acompańamiento al doctorando para que culmine exitosamente la tesis.

Palabras clave: bloques de escritura, doctorado, investigación, tesis. 


\section{The writing blockages in the elaboration of the doctoral thesis}

\section{Summary}

This case study approaches the understanding of the writing blocks that doctoral students experience in the preparation of their thesis. The situations that thesis students incur during the writing process are described and analyzed, in order to later offer an interpretive framework of the factors that affect the blocks. Seven Venezuelan $\mathrm{PhD}$ students were incidentally interviewed. It was found that thesis students present obstacles of a cognitive, behavioral, pedagogical, affective and motivational nature that block them. It is concluded that it is important to offer supervision, motivation and accompaniment to the doctoral student in order to successfully complete the thesis.

Keywords: writing blocks, doctorate, research, thesis. 


\title{
Os bloqueios de escrita na elaboracáo das teses doutorais
}

\begin{abstract}
Resumo
Este estudo de caso aborda a compreensão dos blocos de escrita que os doutorandos vivenciam na elaboração de sua tese. São descritas e analisadas as situaçôes em que os alunos das teses incorrem durante o processo de escrita, para posteriormente oferecer um quadro interpretativo dos fatores que afetam os blocos. Sete estudantes venezuelanos de doutorado foram entrevistados por acaso. Constatou-se que os alunos das teses apresentam obstáculos de natureza cognitiva, comportamental, pedagógica, afetiva e motivacional que os bloqueiam. Conclui-se que é importante oferecer orientação, motivação e acompanhamento ao doutorando para a conclusão com aproveitamento da tese.
\end{abstract}

Palavras chaves: blocos de escrita, doutorado, pesquisa, tese. 


\section{Introducción}

La universidad como centro de producción de conocimientos es una institución que ha sido catalogada como un ente generador de ciencia, que aparte de formar a profesionales, propugna la búsqueda de soluciones a problemas de la sociedad en la cual se inserta el individuo. En los posgrados, entendidos como aquellos estudios de cuarto y quinto nivel en la educación venezolana, se necesita que el aspirante al título de maestría o doctorado, respectivamente, elabore un trabajo de investigación que sirva de elemento de síntesis de su formación teórico-práctica adquirida en los seminarios cursados durante el periodo de escolaridad.

Dicho trabajo se concibe como la tesis y se define como una investigación que recoge las inquietudes científicas del estudiante con miras a generar nuevos hallazgos que favorezcan la comprensión de un problema o tratamiento teórico de un aspecto disciplinar específico. Russell (2013) expone las contradicciones que se presentan en la enseñanza de la escritura a través del currículum y en las disciplinas, alegando que, por lo general, en los programas de las universidades los estudiantes se enfrentan a la imposibilidad de escribir de manera científica, debido al poco desarrollo de las habilidades para la redacción. Cuando un estudiante se encuentra ante el reto de elaborar sus tesis, frecuentemente atraviesa por determinados problemas que lo desalientan y opta por no culminar su trabajo de investigación en el tiempo requerido, volviéndose víctima de la postergación constante, poniendo en riesgo sus estudios de posgrado. $\mathrm{Al}$ respecto, se puede señalar que los bloqueos de escritura suelen ser conductas o limitaciones cognitivas que perjudican el normal desarrollo escritural de la tesis. Bruell (1966), Quaytman (1973), Jacks (1983), Johnstone (1983), Rose (1984) y Boice (1985) estudiaron el fenómeno del bloqueo que ocurre cuando se va a escribir, bien sea un artículo de carácter científico o un texto literario. Los resultados de estas investigaciones revelan la existencia de una serie de factores conductuales, cognitivos y ambientales que impiden la fluidez de las ideas en un escrito y ocasionan que el individuo retrase la función investigadora y codificadora de los pensamientos que desea transmitir. 
Por lo tanto, los bloqueos de la escritura se entienden como aquellos obstáculos que se le presentan a un investigador o tesista, en el proceso de construcción y expresión de las ideas, mostrando dificultades para la traducción de su pensamiento al lenguaje escrito. Zeki (2011) enfatiza que el estudiante, según la poca familiarización que tenga con el proceso de redacción científica, tiende a mostrar miedos e inseguridades que lo conducen a la falta de logro y asertividad para la construcción de un trabajo aceptable desde el punto de vista científico. Una conducta típica que se observa en estas personas es la falta de claridad y organización en la redacción del escrito, que los sume en comportamientos de vulnerabilidad y les hace creer que son incompetentes para la actividad investigativa. Para Carter (2011) escribir va más allá del simple automatismo, implica un proceso de reflexión y autoconocimiento que sirve para que el lector se identifique con las ideas y actúe como un sujeto multiplicador del conocimiento.

Con frecuencia los estudiantes, al momento de elaborar sus tesis de grado muestran desesperación y falta de confianza en que sus escritos cumplan con los criterios metodológicos correspondientes, para ser valorados de manera favorable. En las universidades venezolanas se ha pretendido enseñar a escribir a través del dictado de simples talleres de gramática y ortografía que más que favorecer el pensamiento creativo de los participantes, son la indicación de normas o reglas censoras de las potencialidades escriturales de los estudiantes. Con base en las reflexiones de Ochoa (2009), es pertinente destacar que los docentes se han equivocado al pretender normar la escritura en sus asignaturas, en vez de propiciar el diálogo y la expresión en los universitarios.

Las acciones que se toman en los posgrados para superar las barreras cognitivas y conductuales, que entorpecen la redacción de las tesis, como el dictado de seminarios de investigación, muchas veces representan la exposición de normas o aspectos teóricos sin la correspondiente práctica. Por consiguiente, esta investigación tiene como propósito analizar los bloqueos de escritura que pueden manifestar los estudiantes de doctorado en la elaboración en la tesis, desde un punto de vista descriptivo. 


\section{Fundamentación teórica}

El estudio de la escritura académica, normalmente, se circunscribe dentro del enfoque cognitivo, dado que parte de la atención hasta la autorregulación de las ideas. Sin embargo, Castelló, Bañales y Vega (2010) expresan que la escritura supone no solo un ejercicio de pensamiento y erudición, sino que además involucra la interacción del individuo con su mundo exterior, la percepción que tiene de su entorno, las relaciones que establece con sus pares, el contexto social en el cual se desenvuelve y la identificación cultural que posea.

Carlino (2003) sostiene que la escritura académica adentra al universitario en las actividades de valoración y producción de textos de carácter científico, para que se convierta en un sujeto con capacidad de discernimiento y sentido crítico. Así, una tesis representa una oportunidad para el estudiante de demostrar capacidades investigativas que favorezcan la generación y contrastación de conocimientos, en aras de propiciar nuevos saberes dentro del campo de una disciplina específica.

Ahora bien, la tesis doctoral, como un producto de una investigación consistente, requiere que el estudiante afronte el desarrollo de un trabajo que suponga la aplicación de habilidades de pensamiento superior que ayuden a plasmar un estilo de escritura crítico y creativo. Según Avendaño, Paz y Rueda (2017) los obstáculos para escribir inciden en la redacción del trabajo de investigación, lo cual se ha conocido como el síndrome "todo menos tesis" y podría definirse como el número de estudiantes que no logran culminar sus tesis en el período de tiempo establecido por los programas de formación acreditados por una universidad. Al respecto, una revisión de Valarino (1997) revela que el porcentaje de egresados de doctorados en un período de 9 años en la Universidad Central de Venezuela es de $11,11 \%$, lo que expresa una baja productividad de los posgrados en esa casa de estudios. Gascón (2008) reporta que el porcentaje de graduados en estudios de posgrado es de $13 \%$ en un período de 3 a 5 años.

Honan y Bright (2016) señalan que la redacción de la tesis debe procurar la libertad e independencia de pensamiento del estudiante en pro de fomentar la autonomía, creatividad e iniciativa del individuo, 
para que aporte conocimientos significativos que atraviesen la línea de la mera reproducción de ideas. Diversos autores (Odena y Burgess, 2017; Agné y Mörkenstam, 2018; y Johnson, 2018) refieren que las prácticas de escritura en los doctorados tienen que romper la facilitación de conceptos tradicionales de metodología y ciencias, para que se convierta en un acto de comprensión y proposición de ideas.

Considerando que la tesis representa un esfuerzo mental, los bloqueos de escritura son obstáculos que impiden al estudiante concentrarse y mantener una continuidad o secuencialidad al escribir. Rose (1984) y Boice (1985) definen el bloqueo como un conjunto de inhibiciones o impedimentos que limitan la capacidad de expresar en forma escrita las ideas, debido a la ausencia y falta de estructuración de los conocimientos. Bruell (1966) sugiere que el bloqueo es la inadaptación del escritor a patrones de comportamiento caracterizados por la constancia y sistematicidad. Por ende, estar bloqueado significa la imposibilidad de escribir por carencia de conocimientos, estructuración del texto y la presencia de situaciones que interfieren con el tiempo y dedicación a esta tarea.

Otros autores (Johnstone, 1983; Rennie y Brewer, 1987; Valarino, 1997; y Maher, Feldon, Timmerman y Chao, 2014) señalan que entre los aspectos cognitivos que propician el bloqueo están: incomprensión de las ideas, falta de concentración y fluidez del pensamiento, visión parcelada del conocimiento y deficiencias en lectura y escritura. Entre los aspectos conductuales que abordaron se encuentran: postergación, ansiedad, ambivalencia, impaciencia, aversión a la escritura, estrés, perfeccionismo e inseguridad.

Otro ámbito de interés para el abordaje teórico lo representan los aspectos pedagógicos, afectivos y motivacionales que inciden en los bloqueos de escritura en las tesis, presentes en los trabajos de Cotterall (2011, 2013), Zeki (2011), Difabio (2013) y Johnson (2018). Entre ellos, el control excesivo del tutor; supervisión improvisada; dogmatismo oculto de los profesores; malas prácticas escolares de escritura; presencia de la escritura reproductiva, en vez de la productiva; baja autoestima, minusvalía intelectual; poca persistencia; temor al fracaso; desmotivación; irrelevancia o falta de reconocimiento de la escritura y 
ausencia de impulso e iniciativa. Estos aspectos se convierten en factores que limitan y detienen la elaboración de las tesis doctorales.

Si bien el bloqueo es un fenómeno multifactorial, la ausencia de habilidades de pensamiento superior como el análisis, la síntesis, la argumentación y la metacognición también obstaculiza la escritura. Al respecto, Velásquez, Remolina y Calle (2013) sostienen que los estudiantes universitarios deben adquirir y fortalecer las habilidades de pensamiento que les permitan desarrollar la cognición para comprender lo que se lee y escribe. Por lo tanto, es necesario fomentar estrategias pedagógicas que ayuden al alumno a percibir, observar, entender y comprender el entorno o contexto en que se encuentra.

De lo expuesto por Ochoa y Cueva (2017), es posible señalar que los bloqueos de escritura en la producción de la tesis constituyen dificultades que se presentan para la construcción de las distintas partes de la investigación. Así, el planteamiento de un problema que sea innovador, consistente y sistemático; la interpretación del marco teórico que explique o prediga la realidad abordada; la estructuración de un marco metodológico adecuado a la situación investigada; y la presentación de resultados coherentes, pertinentes y relevantes son procesos que, en la mayoría de los casos, se le dificultan al tesista y lo sumergen en constantes actos de postergación, debido a la falta de claridad en la redacción de las ideas.

Al no encontrar impulso para escribir, el doctorando es proclive a abandonar el trabajo de investigación y desertar del programa de posgrado. La existencia de factores cognitivos que causan el bloqueo propician la aparición de conductas de estrés y ansiedad, lo cual sumado a una formación deficiente en escritura académica durante el pregrado; una situación emocional desequilibrada, caracterizada por inseguridad y miedo a fallar; en estrecha correspondencia con improvisaciones constantes, desinterés y bajo estímulo para escribir, convierten la redacción de la tesis en una actividad limitante y frustrante. 


\section{Metodología}

Esta investigación se inscribe en el paradigma interpretativo; puesto que se partió de la revisión de datos, que fueron analizados para lograr una comprensión del problema abordado (Latorre, del Rincón y Arnal, 1997). En este sentido, se contrastan e interpretan datos reportados por investigadores expertos sobre el tema, a objeto de ofrecer una aproximación al conocimiento de la influencia de los bloqueos de escritura en la redacción de las tesis doctorales. También la investigación es de tipo descriptiva, ya que se caracterizan aspectos de una realidad. Para Perdomo (2005) la descripción parte de registrar, analizar e interpretar los procesos o fenómenos desde una visión del presente. Al respecto, se muestran las respuestas expresadas por los entrevistados en cada una de las categorías de la investigación.

El estudio puso en práctica el diseño de campo, dado que se consideró la recolección de datos directamente del sitio o contexto donde se presenta la situación o problema abordado (Arias, 2012). Para ello, se aplicó una guía de entrevista a un grupo de estudiantes cursantes de un doctorado en educación en una universidad pública venezolana, ubicada en el estado Mérida. Primeramente, se revisó información en diversas fuentes escritas, con la finalidad de revisar el estado actual del conocimiento sobre los bloqueos de escritura en la redacción de las tesis doctorales, tanto a nivel nacional como internacional. Posteriormente, se indagó en los sujetos entrevistados las experiencias de bloqueos que presentaron en la elaboración de la tesis.

En lo metodológico, se aplicó el estudio de casos, considerando que este sirve para describir y analizar entidades educativas (Albert, 2007), por lo cual el estudio se aproxima a las actitudes de un grupo de tesistas y las dificultades de escritura que experimentaron en la redacción de su investigación. La muestra de participantes estuvo conformada por siete estudiantes cursantes de un doctorado en educación en una universidad venezolana, quienes fueron seleccionados de manera incidental, según su accesibilidad y permanencia en los estudios de posgrado en el año 2019. 
Para la recolección y análisis de los datos, se recurrió a una guía de entrevista, estructurada de cinco ítems referidos a los bloqueos de escritura experimentados, las causas y consecuencias de estas dificultades y las estrategias de superación que utilizaron para vencer dichos obstáculos.

Las respuestas obtenidas se procesaron conforme a las siguientes categorías y subcategorías de análisis:

Tabla 1: Matriz de análisis de datos

\begin{tabular}{|c|c|c|}
\hline Categorías & Subcategorías & Indicadores \\
\hline \multirow[t]{2}{*}{$\begin{array}{l}\text { Síntomas de } \\
\text { los bloqueos de } \\
\text { escritura }\end{array}$} & Escriturales & $\begin{array}{l}\text { Repetición constante de ideas, } \\
\text { desorganización en el escrito, monotonía en } \\
\text { la redacción, desestructuración en las ideas, } \\
\text { incomprensibilidad en el texto producido }\end{array}$ \\
\hline & Actitudinales & $\begin{array}{l}\text { Ansiedad, temor o miedo, desesperación, } \\
\text { pesimismo, inconstancia, ambivalencia }\end{array}$ \\
\hline \multirow{2}{*}{$\begin{array}{l}\text { Bloqueos } \\
\text { experimentados } \\
\text { en la redacción } \\
\text { de la tesis }\end{array}$} & $\begin{array}{l}\text { Interpretación y } \\
\text { construcción del } \\
\text { marco teórico }\end{array}$ & $\begin{array}{l}\text { Exposición de ideas argumentativas, formulación } \\
\text { de cuerpo teórico, presentación de la metodología }\end{array}$ \\
\hline & $\begin{array}{l}\text { Presentación } \\
\text { y análisis de } \\
\text { resultados }\end{array}$ & $\begin{array}{l}\text { Contrastación de ideas, redacción de resultados, } \\
\text { elaboración de conclusiones }\end{array}$ \\
\hline \multirow[t]{5}{*}{$\begin{array}{l}\text { Causas de los } \\
\text { bloqueos de } \\
\text { escritura }\end{array}$} & Cognitivas & $\begin{array}{l}\text { Falta de claridad y concentración, poca fluidez de } \\
\text { pensamiento, redacción deficiente, problemas en } \\
\text { lectura y escritura, pocos conocimientos. }\end{array}$ \\
\hline & Conductuales & $\begin{array}{l}\text { Impaciencia, estrés, postergación, } \\
\text { perfeccionismo, aversión a la escritura. }\end{array}$ \\
\hline & Afectivas & $\begin{array}{l}\text { Baja autoestima, miedo a fallar, poca persisten- } \\
\text { cia, presiones laborales. }\end{array}$ \\
\hline & Pedagógicas & $\begin{array}{l}\text { Presiones académicas, dificultades en la relación } \\
\text { con el tutor, control excesivo del tutor, malas } \\
\text { prácticas escolares de escritura, formación } \\
\text { deficiente en investigación }\end{array}$ \\
\hline & Motivacionales & $\begin{array}{l}\text { Falta de impulso para escribir, desmotivación, } \\
\text { falta de iniciativa, poco reconocimiento de la labor } \\
\text { de escritura por parte del tutor }\end{array}$ \\
\hline
\end{tabular}

* Tabla 1 continúa en página siguiente 


\begin{tabular}{lll}
\hline $\begin{array}{l}\text { Consecuencias } \\
\text { de los bloqueos } \\
\text { de escritura }\end{array}$ & Personales & Fracaso, poca capacidad de logro \\
\cline { 2 - 3 } & Académicas & $\begin{array}{l}\text { Prórrogas constantes, deserción o abandono de } \\
\text { los estudios de posgrado }\end{array}$ \\
\hline $\begin{array}{l}\text { Estrategias de } \\
\text { superación }\end{array}$ & $\begin{array}{l}\text { Prácticas de } \\
\text { escritura }\end{array}$ & $\begin{array}{l}\text { Talleres de redacción, ejercicios de composición } \\
\text { de textos argumentativos }\end{array}$ \\
\cline { 2 - 3 } & Acompañamien- & Comunicación y encuentros constantes con el \\
& to académico & tutor, revisiones frecuentes de los escritos \\
\hline
\end{tabular}

Elaboración propia, a partir de la guía de entrevista aplicada.

\section{Resultados}

Tras la aplicación de una guía de entrevista a cursantes de un doctorado en educación, en una universidad pública venezolana, se lograron obtener los siguientes resultados, según se presentan por categorías de análisis:

\section{Sintomas de bloqueos de escritura}

La mayoría de los entrevistados reportó que comenzó a sentirse bloqueado en la aparición recurrente de sentimientos de ansiedad, pesimismo, miedo, temor, ambivalencia e inconstancia en el trabajo de escritura de las diferentes partes de la tesis doctoral. En diferentes oportunidades señalaron que sus escritos eran repetitivos, monótonos y desorganizados.

Me he sentido agobiado por la escritura de mi tesis, al punto de verme frustrado en la tarea de redacción. En varios momentos me sentí malhumorado y con un gran sentimiento de fracaso; pues no lograba concentrarme y siempre prefería hacer otra cosa que redactar mi trabajo. (Entrevistado No 3)

Se aprecia la presencia de síntomas que evidencian bloqueos de escritura, tanto desde el punto de vista escritural como actitudinal que sumergen al individuo en situaciones de pesimismo e inconstancia en el trabajo. 


\section{Bloqueos experimentados en la redacción de la tesis}

La mayoría de los entrevistados señaló que los bloqueos de escritura que experimentaron estuvieron caracterizados por la redacción de la parte expositiva y argumentativa de los diferentes apartados de la tesis doctoral, específicamente en el planteamiento del problema, la presentación de los resultados y la elaboración de conclusiones. Una de las respuestas dadas, expresa lo siguiente:

Me era difícil plantear el problema porque no tenía en claro si el tema trabajado realmente constituía un verdadero problema de investigación. También me tuve que enfrentar a mi falta de sentido crítico que el tutor me exigía en la presentación de los resultados. (Entrevistado No 2)

Se evidenciaron dificultades de escritura, caracterizadas por la falta de claridad y pertinencia en el contraste de los resultados obtenidos con lo esbozado en la fundamentación teórica de la tesis, lo cual es un error frecuente que impide al estudiante elaborar un trabajo con consistencia científica. Al no contar con habilidades de pensamiento superior se dificulta la crítica, argumentación, reflexión y reconstrucción de ideas.

Otro de los entrevistados reveló que:

Empleaba mucho tiempo en contrastar los resultados de mi investigación con los reportes de otros estudios y con las bases teóricas; pero no lograba escribir de manera pertinente. Más bien terminaba reafirmando lo que decían los autores revisados y abandonaba mis propias ideas. (Entrevistado No 4)

\section{Causas de los bloqueos de escritura}

De acuerdo con los resultados obtenidos, la gran mayoría de ellos señaló que sus bloqueos de escritura radicaban en la falta de habilidades para la redacción, confusión en la presentación de las ideas, ansiedad, impaciencia, estrés, postergación constante, miedo a fallar y la ausencia de experiencias de escritura creadoras, entre otros aspectos, 
los cuales constituyeron limitaciones para culminar la tesis. El resto de los participantes mencionó que las presiones académicas y laborales, la poca retroalimentación por parte del tutor, aunado todo a la inseguridad de sus conocimientos en metodología de la investigación, representaron una dificultad que les ocasionó bloqueos constantes en la producción de los escritos.

No pude seguir escribiendo mi tesis porque la presión de mi tutor, constantemente, me impedía concentrarme. Por varios meses me sentí impotente ante la tarea de escritura. Aparte de ello, mi poca experiencia en la escritura académica, la inseguridad ante la manera correcta de presentar mis argumentos y la poca gratificación hacia el proceso de escritura no me convencían de seguir haciendo la tesis. (Entrevistado No 5)

Este testimonio de uno de los entrevistados pone de manifiesto que los bloqueos, en la redacción de la tesis doctoral, ocurren desde diversas vertientes que se asocian con factores cognitivos, conductuales, afectivos, pedagógicos y motivacionales. Así, escribir la tesis puede convertirse en una tarea ardua sujeta a constantes presiones que no siempre van a permitirle al doctorando cumplir con la elaboración de su trabajo de investigación.

\section{Consecuencias del bloqueo}

La mayoría de los entrevistados reportó que la consecuencia directa de los bloqueos en la escritura de la tesis doctoral es el abandono de la investigación y el fracaso en la obtención del título doctoral. Según las respuestas recogidas en este aspecto, la solicitud de prórrogas ante las comisiones de tesis, se debe a que el doctorando se siente inseguro e incapaz de cumplir con los parámetros académicos previstos en los programas de posgrado. Otra de las consecuencias previsibles es la deserción o retiro de los posgrados como resultado de un sentimiento de minusvalía ante el reto de elaborar un trabajo escrito de elevada envergadura.

El siguiente testimonio permite observar que el doctorando tiende a abandonar la tarea de escritura como consecuencia de sentirse bloqueado: 
Al no poder continuar escribiendo mi tesis, opté por darle largas que, a veces, se traducían en la solicitud de constantes prórrogas, en las cuales no hacía nada más que esperar la siguiente prórroga. Este círculo vicioso me perjudicó enormemente y me empujó a abandonar el posgrado por un tiempo. (Entrevistado No 1)

Ciertamente, el abandono de la escritura de la tesis es una de las consecuencias de sentirse bloqueado, al verse imposibilitado de cumplir con las exigencias del posgrado.

Otro de los entrevistados indicó:

Padecí los efectos de estar bloqueado en la escritura de manera constante, y noté este comportamiento frecuente cuando apenas comenzaba a escribir alguno de los párrafos y abandonaba a cada rato esta tarea. Eso me hacía pedir prórrogas que, a veces, las solicitaba por mera inercia. (Entrevistado No 3)

Ambos testimonios permiten inferir que los tesistas al no poder culminar el trabajo de investigación por distintas limitaciones incurren en el abandono del proceso de escritura, lo cual puede generarles sensaciones de fracaso.

\section{Estrategias de superación del bloqueo}

La totalidad de los entrevistados reportó que las estrategias que les ayudaron a vencer los bloqueos de escritura estuvieron constituidas por actividades de acompañamiento por parte del tutor y los profesores del posgrado, además de la capacidad de reflexionar sobre el propio pensamiento que les permitió autorregular la escritura. El siguiente testimonio permite comprender cómo afrontaron estas dificultades los entrevistados:

Mi problema de los bloqueos constantes logré superarlos gracias a la ayuda de un asesor externo en metodología y escritura académica que me motivaba a escribir y vencer el miedo a fracasar. Esto me ayudó mucho, pues recuperé mi confianza y 
seguridad y la labor de escritura se hizo más llevadera. (Entrevistado No 5).

Como bien se aprecia, la motivación forma parte de una de las estrategias que pueden contribuir a superar los bloqueos de escritura, dado que implica permitirle al estudiante vencer los propios obstáculos mentales que, a veces, son más limitantes que aquellas de carácter académico y administrativo que se observan en algunos programas de posgrado.

Otra de las respuestas expresa:

Mi tutor fue quien me impulsó a culminar mi tesis doctoral. Sus consejos y constantes correcciones me permitieron finalizar mi trabajo de manera exitosa (...) en el sentido de que yo podía pensar por mí mismo en mis errores de escritura y corregirlos. (Entrevistado No 2)

A juzgar por la valoración que el entrevistado realiza de su relación con el tutor, se comprende que el apoyo mutuo entre tesista y tutor fortalece y conduce a la culminación del trabajo, en aquellos momentos de ansiedad o pérdida de autoconfianza, aunado a la metacognición cuando se está escribiendo que favorece la autorregulación del pensamiento.

\section{Discusión}

A través de la interpretación de los resultados de la investigación se pone de manifiesto que los síntomas del bloqueo pueden evidenciarse en la presencia de sentimientos de pesimismo, ansiedad o temor del estudiante ante la elaboración de la tesis. La escritura monótona, repetitiva y desorganizada que no refleja con claridad las ideas, también constituyen signos de estar bloqueado. Las respuestas de los entrevistados describen cómo se presenta la imposibilidad de culminar la tesis, lo cual coincide con las apreciaciones de Valarino (1997), Cotterall (2013) y Maher, Feldon, Timmerman y Chao (2014), quienes revelan la manera en que se evidencian las inhibiciones cuando se intenta escribir. 
Los bloqueos que experimentaron los entrevistados en la producción escrita denotan que la interpretación y reinterpretación de los componentes teóricos de una investigación requieren de un esfuerzo intelectual que se obtiene mediante un proceso de búsqueda y reflexión continua a partir de la información. Asimismo, la discusión de los resultados es una tarea detallada y sistemática que implica la contrastación de lo que se ha escrito sobre un problema de investigación y los hallazgos que se logren en la tesis. Esta situación coincide con lo expresado por Difabio (2013) y Ochoa y Cueva (2017) en cuanto a la inclinación de los estudiantes por reproducir ideas, en detrimento de la construcción de las mismas.

Las múltiples causas de bloqueos, reportadas por los sujetos abordados en el estudio muestran que los factores cognitivos y conductuales son decisivos en las dificultades de redacción; aunado la falta de experiencias constructivas de escritura en la formación académica, deficiente manejo del estrés, las presiones, el fracaso y el poco estímulo e impulso para culminar la tesis. Tales factores constituyen, según lo referido por Johnstone (1983), Rennie y Brewer (1987), Valarino (1997) Cotterall (2011), Zeki (2011) y Johnson (2018), obstáculos individuales que si se conjugan pueden convertirse en inhibidores de la escritura.

Con relación a las consecuencias de los bloqueos que experimentaron los doctorandos, se aprecia que el abandono de la investigación y la sensación de fracaso constituyen efectos de los obstáculos de escritura. Esta conducta concatena con lo expresado por Ochoa y Cueva (2017) al describir los sentimientos de angustia y pesimismo que manifiestan los tesistas durante el proceso de redacción, lo cual puede conducir a la deserción del programa de doctorado, al no poder enfrentar el reto de escribir desde una perspectiva científica (Valarino, 1997 y Carlino, 2003).

En cuanto a las estrategias de superación, los tesistas reportaron que el acompañamiento, la motivación, el apoyo del tutor y la autorregulación de la escritura son acciones que ayudan a obtener el éxito en la culminación de la investigación. Estas opiniones coindicen con lo que se infiere de Odena y Burgess (2017), Agné y Mörkenstam (2018) y Johnson (2018); puesto que un proceso de facilitación adecuado entre 
tesista y tutor que establezca retroalimentaciones productivas, en un marco de colaboración y apoyo, incide en la finalización de la tesis. Adicionalmente, la metacognición tal como lo establecen Velásquez, Remolina y Calle (2013), permite autorregular el pensamiento y forman parte de las habilidades que se deben desarrollar en la escritura académica (Castelló, Bañales y Vega, 2010).

Como bien se ha analizado, los bloqueos en la redacción de las tesis doctorales son obstáculos, de diferente naturaleza, que inhiben el proceso de escritura en el estudiante, experimentando una serie de situaciones individuales, como resultado de la imposibilidad de culminar el trabajo de investigación. Estas situaciones comprenden la existencia de sentimientos de frustración ante el desafío de escribir de manera científica con la claridad, pertinencia y sistematicidad que se requiere en la redacción de un producto que refleje la capacidad de expresarse en forma académica. Si bien la tesis constituye un trabajo de procesamiento de la información, a veces, no se está exento de limitaciones que detienen el proceso de escritura, necesitando la aplicación de estrategias que permitan reflexionar para repensar en la pertinencia y adecuación de las propias ideas que se plasman en el texto producido.

Los resultados también ponen de manifiesto que los entrevistados fueron conscientes de que sus textos iniciales no reunían las condiciones de sistematicidad y claridad necesarias, por lo que fueron capaces de autorregular y controlar el proceso de escritura, superando las limitaciones que les impedían, en algunos casos, la fluidez de pensamiento. De esta manera, es importante que el tesista comprenda que escribir representa una lucha contra sus propias convicciones y miedos para expresar su pensamiento, lo cual requiere de atreverse a revelar sus ideas, teniendo consciencia de que va a ser escrutado y juzgado para que sea reconocido como un investigador competente en el ámbito académico.

\section{Conclusiones}

El análisis de los resultados del estudio de casos realizado, evidencia que los bloqueos de escritura obstaculizan la producción de co- 
nocimiento en los tesistas doctorales, al no poder vencer de manera adecuada los factores cognitivos, conductuales, pedagógicos, afectivos y motivacionales que inhiben las potencialidades para procesar y presentar información escrita. La falta de habilidades de pensamiento representa un inconveniente que dificulta la creación de textos en los estudiantes, debido a malas prácticas de escritura académica, que no favorecen la metacognición como proceso que permite repensar lo que se escribe.

La aparición de síntomas de bloqueo puede manifestarse, tanto desde el punto de vista actitudinal como escritural y el tesista puede estar experimentando esta situación sin tener conciencia de ello, anclándose en la postergación constante o abandonando la actividad de investigación. Una tesis, como producto sólido de investigación, parte de la búsqueda y revisión de información y finaliza con el sometimiento a contraste de los hallazgos obtenidos con los fundamentos teóricos que se analicen. De allí, la importancia de que en las diferentes partes del trabajo el estudiante sea capaz de conciliar la espontaneidad de su propio pensamiento con la rigurosidad y sistematicidad que suele utilizarse en la redacción de un trabajo científico.

Las causas del bloqueo revelan las falencias individuales, académicas y escriturales que los tesistas de doctorado exhiben en el proceso de producción escrita. Se supone que un doctorando debe haber adquirido, en su formación previa, habilidades de pensamiento superior que le permitan autorregular su propia escritura. Sin embargo, tal situación indica la necesidad de mejorar las competencias de los estudiantes de pregrado y posgrado universitario, en cuanto a la práctica continua de la escritura académica, procurando que no solo sea un simple ejercicio recordatorio de reglas de redacción, sino que realmente facilite conocimientos para poder elaborar un texto científico.

Una de las consecuencias más graves de que un tesista doctoral presente bloqueos de escritura es el abandono del programa de posgrado. La falta de superación de los obstáculos que inhiben su capacidad para expresar sus pensamientos de manera escrita, lo puede sumergir en situaciones de minusvalía intelectual que, si no logra vencer apropiadamente, le convierten en un individuo con serias limitaciones para 
actuar como un investigador competente. Por ende, se requiere de la aplicación de estrategias que coadyuven en la superación de los bloqueos, mediante el acompañamiento académico del tesista que muestra dificultades para la culminación de su investigación. Una relación adecuada y productiva entre tesista y tutor, aunado a una retroalimentación clara de los escritos que se presenten, perfilan el éxito en la finalización de la tesis.

En vista de lo antes expuesto, este estudio representa un abordaje descriptivo de los bloqueos que experimentan los tesistas doctorales en el cumplimiento del principal requisito de grado, como lo es la realización de un trabajo de investigación competente, que dé indicios de las capacidades para procesar y generar información de carácter científico. No obstante, el estudio puede ser replicado en otras universidades o programas de posgrado e incluirse la aplicación de estrategias de afrontamiento de los bloqueos de escritura, lo cual constituirá una experiencia que permitirá conocer los logros o avances que obtengan los tesistas. 


\section{Referencias}

Agné, H. y Mörkenstam, U. (2018). Should first-year doctoral students be supervised collectively or individually? Effects on thesis completion and time to completion [Revista en línea]. Higher Education Research \& Development, 37(4), pp. 669682. https://doi.org/10.1080/07294360.2018.1453785

Albert, M. (2007). La investigación educativa, claves teóricas. España: Mc Graw Hill.

Arias, F. (2012). El proyecto de investigación, introducción a la metodología cientifica ( $6^{\mathrm{a}} \mathrm{ed}$.). Caracas: Espíteme.

Avendaño, W., Paz, L. y Rueda, G. (2017). Dificultades en la escritura académica y funciones cognitivas: revisión [Revista en línea]. Sophia, 13(1), pp. 132-143. http://dx.doi. org/10.18634/sophiaj.13v.1i.457

Boice, R. (1985). Cognitive component of blocking [Revista en línea]. Written Communication 2, 91-104. Recuperado de: http:// journals.sagepub.com/doi/10.1177/0741088385002001006

Bruell, E. (1966). How to block the reading blocks? Read! [Revista en línea]. Peabody Journal of Education, 44(29), pp. 114-117. https://doi.org/10.1080/01619566609537405

Carlino, P. (2003). Alfabetización académica: Un cambio necesario, algunas alternativas posibles [Revista en línea]. Educere, 6(20), pp. 409-420. Recuperado de: http://www.redalyc.org/articulo. oa?id=35662008

Carter, S. (2011). Doctorate as genre: supporting thesis writing across campus [Revista en línea]. Higher Education Research \& Development, 30(6), pp. 725-736. https://doi.org/10.1080/07 294360.2011 .554388

Castelló, M., Bañales, G. y Vega, N. (2010). Enfoques en la investigación de la regulación de escritura académica: Estado de la cuestión [Revista en línea]. Electronic Journal of Research in Educational Psychology, 8(3), pp. 1253-128. Recuperado de: http://www.redalyc.org/articulo.oa?id=293122000015 
Cotterall, S. (2011). Doctoral students writing: where's the pedagogy? [Revista en línea]. Teaching in Higher Education, 16(4), pp. 413-425. https://doi.org/10.1080/13562517.2011.560381

Cotterall, S. (2013). More than just a brain: emotions and the doctoral experience [Revista en línea]. Higher Education Research and Development, 32(2), pp. 174-187. https://doi.org /10.1080/07294360.2012.680017

Difabio de Anglat, H. (2013). Evaluación de las concepciones de escritura académica en doctorandos en educación [Revista en línea]. Revista Electrónica Actualidades Investigativas en Educación, 13(3), pp. 1-21. Recuperado de: http://www. redalyc.org/articulo.oa?id $=44729878011$

Gascón, Y. (2008). El síndrome todo menos tesis “TMT” con factor influyente de la actividad investigativa [Revista en línea]. Copernico, 4(7), pp. 32-43. Recuperado de: http://copernico. uneg.edu.ve/numeros/c09/c09_art05.pdf

Honan, E. y Bright, D. (2016). Writing a thesis differently [Revista en línea]. International Journal of Qualitative Studies in Education, 29(5), pp. 731-7343. https://doi.org/10.1080/09518398.201 6.1145280

Jacks, P. (1983). The ABCs of ABDs: a study of incomplete doctorates [Revista en línea]. Improving College and University Teaching, 31(2), 74-81. Recuperado de: https://www.tandfonline.com/ doi/abs/10.1080/00193089.1983.10533805

Johnson, E. (2018. The doctoral writing conversation: establishing a generic doctoral writing programme [Revista en línea]. Open Review of Educational Research, 5(1), pp. 1-12. https://doi.org/ 10.1080/23265507.2017.1419439

Johnstone, A. (1983). The Writer's Hell: Approaches to Writer's Bloc [Revista en línea]. Journal of Teaching Writing, 2(2), 155165. Recuperado de: https://journals.iupui.edu/index.php/ teachingwriting/article/view/677/652

Latorre, A., del Rincón, D. y Arnal, J. (1997). Bases metodológicas de la investigación educativa. Barcelona: Hurtado Ediciones. 
Maher, M., Feldon. D, Timmerman, B. y Chao, J. (2014). Faculty perceptions of common challenges encountered by novice doctoral writers [Revista en línea]. Higher Education Research and Development, 33(4), pp. 699-711. https://doi.org/10.1080 /07294360.2013.863850

Ochoa, L. (2009). La lectura y la escritura en las tesis de maestría [Revista en línea]. Forma y Función, 22 (2), pp. 93119. Recuperado de: http://www.redalyc.org/articulo. oa?id=21916691005

Ochoa, L. y Cueva, A. (2017). El bloqueo en el proceso de elaboración de una tesis de maestría: angustias y desazones percibidas por sus protagonistas [Revista en línea]. Lenguaje, 45 (1), pp. 61-87. Recuperado de: http://www.scielo.org.co/ pdf/leng/v45n1/0120-3479-leng-45-01-00061.pdf

Odena, O. y Burgess, H. (2017). How doctoral students and graduates describe facilitating experiences and strategies for their thesis writing learning process: a qualitative approach [Revista en línea]. Studies in Higher Education, 42(3), pp. 571590. https://doi.org/10.1080/03075079.2015.1063598

Perdomo, R. (2005). Metodología de la investigación jurídica. Mérida, Venezuela: Consejo de Publicaciones de la Universidad de Los Andes.

Quaytman, W. (1973). Psychotherapist's Writing Block [Revista en línea]. Journal of Contemporary Psychotherapy, 4(1), 53-57. Recuperado de: https://link.springer.com/article/10.1007/ BF02110276

Rennie, D. y Brewer, L. (1987). Grounded Theory of Thesis Blocking [Revista en línea]. Teaching of Psychology, 14(1), pp. 10-16. Recuperado de: http://journals.sagepub.com/doi/pdf/10.1207/ s15328023top1401_2

Rose, M. (1984). Writer's Block: The Cognitive Dimension. Studies in Writing \& Rhetoric [Libro en línea]. Illinois, USA: Editorial Carbondale, Southern Illinois University Press. Recuperado de: https://files.eric.ed.gov/fulltext/ED248527.pdf 
Russell, D. (2013. Contradictions regarding teaching and writing (or writing to learn) in the disciplines: What we have learned in the USA [Revista en línea]. Revista de Docencia Universitaria, 11 (1), pp. 161-181. Recuperado de: http://www.red-u.net/

Valarino, E. (1997). Tesis a tiempo. Caracas: Equinoccio

Velásquez, B. Remolina, N, y Calle, M. (2013). Habilidades de pensamiento como estrategia de aprendizaje para los estudiantes universitarios. Revista de Investigaciones UNAD, 12(2), pp. 23-41.

Zeki, K. (2011). The Effects of Various Variables on University Students' Writer's Block Levels [Revista en línea]. The Antopologist, 21(1-2), pp.311-322. https://doi.org/10.1080/09 720073.2015 .11891820 